\title{
Autism Spectrum Disorder and COVID-19: Helping Caregivers Navigate the Pandemic
}

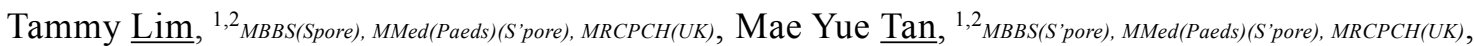
Ramkumar Aishworiya, ${ }^{1,2}$ MBBS(S'pore), MMed(Paeds)(S'pore), MRCPCH (UK), Ying Qi Kang, ${ }^{1,2}$ MBBS(S'pore), MMed(Paeds)(S'pore), MRCPCH (UK)

The coronavirus disease 2019 (COVID-19) pandemic has disrupted societies globally. As of 11 May 2020, 53 children have been infected with COVID-19 in Singapore (Ministry of Health, Singapore, unpublished data). Children generally have mild disease, ${ }^{1}$ although there is emerging literature on paediatric multisystem inflammatory syndrome temporally associated with COVID-19. Less well reported is the impact of COVID-19 on the daily lives and psychology of children. This article highlights the implications of COVID-19 on an especially vulnerable population of children - children with autism spectrum disorder (ASD). ASD is a developmental disorder characterised by impaired social communication, language, and restricted stereotypic behaviour with rigidities. ASD is prevalent. It is estimated that 50,000 individuals are affected by ASD in Singapore. ${ }^{2}$ Healthcare professionals are likely to encounter persons with ASD in their routine practice. Based on our experience in our developmental and behavioural paediatrics practice, caregivers experience significant challenges in managing children with ASD during this pandemic. Children with ASD are having difficulties adjusting to change and feeling anxious, whilst caregivers themselves are worried and fatigued. This article aims to be a resource for healthcare professionals in supporting these families. Strategies depicted are based on behavioural management principles used for ASD - promoting structure, use of antecedent strategies and positive reinforcement of desirable behaviours.

\section{Sharing of Information}

Children with ASD are cognisant about changes in their environment. A trusted caregiver should explain these changes to the child in a developmentally appropriate manner and focus on providing reassurance for the child. As these children have relative visual strengths, the use of visual supports ${ }^{3}$ may help the child understand the situation better. For those who are younger, have lower language or cognitive abilities, videos and social stories with pictures will be helpful. For children who use language, caregivers can start by asking their child what he/she already knows, what questions he/she may have, and then supplement or correct their understanding accordingly. The dialogue should be direct as these children might not be able to infer from vague messages. For example, instead of saying, "There is a virus and it's dangerous to go outside", consider saying "Staying at home will reduce the chances of getting sick". Knowledge sharing should happen regularly for caregivers to address new concerns that may arise. Exposure to COVID-19 related media exposure should be monitored and restricted.

\section{Coping with Change}

Individuals with ASD prefer routines and may have difficulty adjusting to changes. However, during this period, changes are common. To help children cope with change, caregivers should maintain a child's daily routine. When this is not possible, pre-empt the child about any change and adopt a new routine while allowing time for the child to adapt to it. A visual schedule of the day's routine will help the child know what to expect and transit between activities. Timers can further provide children with visual and auditory cues. Allocated time and space for various activities (e.g. mealtime, learning, play) will help the child understand specific events better. For less verbal children, the TEACCH (Treatment and Education of Autistic and Communication related handicapped Children) method of using actual items to help children conceptualise a routine is helpful. Empower the child by giving them control over small decisions, like choosing a preferred type of meal. Pique their

\footnotetext{
${ }^{1}$ Khoo Teck Puat-National University Children's Medical Institute, National University Hospital, Singapore

${ }^{2}$ Department of Paediatrics, Yong Loo Lin School of Medicine, National University of Singapore, Singapore

Address for Correspondence: Dr Tammy Lim, Department of Paediatrics, Division of Developmental and Behavioural Paediatrics, Khoo Teck Puat National University Children's Medical Institute, National University Hospital, 1E Kent Ridge Road, NUHS Tower Block Level 12 , Singapore 119228. Email: tammy_sh_lim@nuhs.edu.sg
} 
motivation to new measures like hand washing and mask wearing by proposing these as methods they can protect themselves with.

\section{Adjusting to New Routines}

New routines like hand washing, use of masks, social distancing, restricted outdoor access and home-based learning are new norms. Children with ASD may have difficulties grasping the reason for these practices and in understanding that his/her caregiver is now also a teacher. In addition, home environments may not be conducive for learning if they are too sensory stimulating or if siblings are distracting.

Caregivers should introduce new routines in a positive manner. It is more effective to tell children what to do, than what not to do. Caregivers should use instructions like "hands by your side", instead of "don't touch your face". For younger or less verbal children, keep the message simple by using keywords, direct demonstration or visual aids. For example, to promote hand hygiene, the caregiver can use a hand gesture indicating hand washing coupled with a verbal cue. To help children maintain adequate distance from others, teach them to stretch out both their arms to use that as a physical boundary. Use of social stories can aid in explaining these concepts. Caregivers could use a reward system to reinforce compliance to desirable behaviours.

\section{Maintaining Connections with Loved Ones}

Restrictions in movement between households can result in children being unable to interact with familiar caregivers. The absence of familiar caregivers like teachers and grandparents can make children feel anxious. Caregivers could help families maintain social connections via telephone or video calls, and use a visual countdown to represent when these reunions can take place in-person.

\section{Self-care of Caregivers}

Caregivers of children with ASD experience more stress. ${ }^{4}$ Further, for a parent raising a child with ASD, the risk of self-neglect is higher. Parents often postpone their own needs for their children and family. With increased parenting, coupled with work from home demands during this pandemic, this stress is likely to be exacerbated. Parents of children with ASD already feel socially isolated at baseline; social distancing during this pandemic could aggravate the sense of loneliness. It is important for caregivers to take care of themselves. A caregiver's health and well- being is directly related to the quality of care they can provide their children. Specific to COVID-19, parental anxiety can also lead to children worrying more.

Caregivers need to first ensure that they are caring for themselves physically with adequate nutrition and rest. Next, they need to practice emotional self-care by acknowledging and addressing emotional burdens they are experiencing. Thirdly, they should practice spiritual self-care, through a process of doing something they enjoy. This can be by taking scheduled short breaks from parenting, or making time for hobbies. Using hobbies as activities which the parent and child can do together may be helpful. Caregivers should not feel guilty for offering their children some screen time during this period. Actively seeking help for both emotional and tangible support (for example, caring for the child) is encouraged, as is seeking help from professionals when feeling overwhelmed.

Risk factors for increased stress include: having more than one child with a disability, disadvantaged socioeconomic status, children with severe ASD who require constant supervision and assistance for activities of daily living, children with disrupted sleep, and caregivers with little social support. ${ }^{5}$ Using the resources in Figure 1, physicians can reach out to these families and offer support.

\section{Managing the Child's Anxiety}

Children with ASD are are at a higher risk of having anxiety. ${ }^{6}$ Language impairment may limit communication of emotions and make their anxiety harder to detect. Anxiety is likely to be exacerbated in a pandemic setting. ${ }^{7}$ Anxiety in these children may manifest as tantrums, challenging behaviours,

1. Enabling guide - Latest information on Coronavirus Disease 2019

2. NUH COVID-19 Resources for Parents and Caregivers

3. $\mathrm{MOH}$ Special Care Kit - Resources to support individuals with special needs for COVID-19 related healthcare encounters

4. Let's fight COVID-19 together with Superhero me

5. KKH COVID-19 Care Resources

6. Singapore National Care Hotline

7. Tinkle Friend Hotline by the Singapore Children's Society

Fig. 1. COVID-19 community resources for persons with special needs in Singapore 
distractedness, separation anxiety, trouble eating or sleeping, behavioural regression and/or increase in repetitive behaviours. These changes are reactions to disruptions faced, and are likely transient. Children should be encouraged to express their feelings verbally, or through writing, drawing, or play. Caregivers should be good listeners and avoid 'playing down' a child's fears as this can exacerbate the anxiety. Older children with ASD may benefit from mindfulness practice. ${ }^{8}$ This can include being mindful of their behaviours, and learning coping and calming skills. Parents can help by modelling these practices. When required, help from psychologists/ psychiatrists should be sought. Cognitive behavioural therapy ${ }^{9}$ is a widely accepted psychological approach for treating anxiety in children who use words to communicate. In some cases, medication can be helpful.

\section{Conclusion}

Healthcare professionals should be aware that caregivers of children with ASD face additional challenges during this pandemic. We have outlined strategies that can provide support to these families. In view of the immense stress some families endure, healthcare professionals should be vigilant about family violence, to enquire about family stress levels and make violence screening a part of every assessment. Look for mood disorders in caregivers and unusual fearfulness in children. Reaching out to vulnerable families proactively when there are no appointments in the near future can help avert violence. With know-how, healthcare professionals can help families with persons with ASD better manage this challenging COVID-19 period.

\section{Acknowledgements}

We would like to thank Dr Dimple Rajgor for helping with formatting and submission of the manuscript for publication.

\section{REFERENCES}

1. Goh KJ, Choong MC, Cheong EH, Kalimuddin S, Duu Wen S, Phua GC, et al. Rapid progression to acute respiratory distress syndrome: Review of current understanding of critical illness from COVID-19 infection. Ann Acad Med Singapore 2020;49:108-18.

2. Autism resource centre (Singapore). Prevalence of autism in Singapore 2016. Available at: https://www.autism.org.sg/living-withautism/prevalence-of-autism-in-singapore. Accessed on 1 June 2020.

3. Rutherford M, Baxter J, Grayson Z, Johnston L, O'Hare A. Visual supports at home and in the community for individuals with autism spectrum disorders: A scoping review. Autism 2020;24:447-69.

4. Schieve LA, Blumberg SJ, Rice C, Visser SN, Boyle C. The relationship between autism and parenting stress. Pediatrics 2007;119:S114-21.

5. Catalano D, Holloway L, Mpofu E. Mental health interventions for parent carers of children with autistic spectrum disorder: Practice guidelines from a critical interpretive synthesis (CIS) systematic review. Int J Environ Res Public Health 2018;15:341.

6. Hollocks MJ, Lerh JW, Magiati I, Meiser-Stedman R, Brugha TS. Anxiety and depression in adults with autism spectrum disorder: a systematic review and meta-analysis. Psychol Med 2019;49:559-72.

7. Taha S, Matheson K, Cronin T, Anisman H. Intolerance of uncertainty, appraisals, coping, and anxiety: the case of the 2009 H1N1 pandemic. Br J Health Psychol 2014;19:592-605.

8. Ridderinkhof A, de Bruin EI, Blom R, Bögels SM. Mindfulnessbased program for children with autism spectrum disorder and their parents: direct and long-term improvements. Mindfulness (NY) 2018;9:773-91.

9. Ho CS, Chee CY, Ho RC. Mental health strategies to combat the psychological impact of COVID-19 beyond paranoia and panic. Ann Acad Med Singapore 2020;49:155-60. 